\title{
The concentration of radon in buildings in Central-Southern Italy: experimental campaigns \& correlation modelling
}

\author{
O. Barra ${ }^{1}$, A. Barra ${ }^{2}$, S. Cimbalo ${ }^{3}$, A. Cuiuli ${ }^{2}$, L. Franceschi ${ }^{2}$, P. \\ Ionta $^{3} \&$ D.E. Sytnikov ${ }^{2}$ \\ ${ }^{I}$ University of Calabria, Physics Dept., Cosenza, Italy. \\ ${ }^{2}$ LIFE Ltd, Laboratories for Information Food and Energy, Rome, Italy. \\ ${ }^{3}$ Associazione EDENET, Post-graduate School on Energy and \\ Environment, Palazzo Cosentini, Feroleto Antico, Catanzaro, Italy.
}

\section{Abstract}

The paper aims to make available a wide set of experimental results deriving from a 30-months measurement campaigns (1999-2001) on going in Central and Southern Italy, capable of detecting the indoor radon concentration levels in some 1000 buildings. The research is supported by LIFE, Calabria Regional Authority, Italian Ministry of University and Scientific Research and several Builders Association. The methodology is based on the use of high quality portable ionisation chambers, having an error less than $5 \%$ and a certified calibration better than $1 \%$. The radon emitted $\alpha$-particles are detected every 10 minutes - for at least 5 days for each site - jointly with the ambient temperature, relative humidity and pressure, with a continuous check of the Data QA and System QA. An on-purpose developed on-line software is capable to evaluate relocation markers, noise reduction, time distribution, smoothing and synchronisation, as well as radium progeny, exhalation rate and equilibrium factors. The paper, because of such a huge number of experimental data, shows reliable correlation formulas between radon concentration levels and types of soil, ambient parameters and building features. The results show that some 15 . $20 \%$ of buildings exhibit radon concentration values in the range which is dangerous to health $\left(>400 \mathrm{~Bq} / \mathrm{m}^{3}\right)$ and up to $50 \%$ of building in the range in which health is at risk $\left(150-400 \mathrm{~Bq} / \mathrm{m}^{3}\right)$. 


\section{Environmental Health Risk}

\section{Radon in buildings: sources}

Human beings as well as any other living body, is exposed to ionising radiations coming from different natural sources. The typical relevant ranges of the EBR (Effective Biological Relative Dose) can be summarized as follows:

Table 1. Typical range of the EBR due to the various sources.

\begin{tabular}{lccl}
\hline & $\begin{array}{c}\text { WORLD } \\
\text { AVERAGE }(\mathrm{mSv} / \mathrm{y})\end{array}$ & $\begin{array}{c}\text { RANGE } \\
\mathrm{mSv} / \mathrm{y}\end{array}$ & \multicolumn{1}{c}{ NOTES } \\
\hline $\begin{array}{l}\text { Outdoor Radiation } \\
\text { Cosmic Rays }\end{array}$ & 0.36 & $0.30-2.0$ & $\begin{array}{l}\text { Constant at sea level Growing with } \\
\text { altitude Doubling at } 1500 \mathrm{~m}\end{array}$ \\
$\begin{array}{l}\text { Terrestrial Sources } \\
\begin{array}{l}\text { Indoor Radiation } \\
\text { (coming from the building materials } \\
\text { and soil below them) }\end{array}\end{array}$ & 0.41 & $0.20-1.0$ & $\begin{array}{l}\text { Due to U-238, Th-232, K-40 present in } \\
\text { soil Greater in igneous, P and Al rocks } \\
\text { K-40 }\end{array}$ \\
$\begin{array}{l}\text { Ra-226, Rn-222 } \\
\text { and progeny }\end{array}$ & 0.18 & $0.10-0.2$ & $\begin{array}{l}\text { materials and building structure and } \\
\text { typology }\end{array}$ \\
\hline $\begin{array}{l}\text { TOTAL; } \\
\text { equivalent to: }\end{array}$ & 1.44 & $0.30-80.0$ & $\begin{array}{l}\text { Depending on soil, buil. materials and } \\
\text { building structure and typology }\end{array}$ \\
\hline
\end{tabular}

Today it is worldwide accepted that a level $>400 \mathrm{~Bq} / \mathrm{m}^{3}$ is certainly highly dangerous for human health; a level between 100 and $400 \mathrm{~Bq} / \mathrm{m}^{3}$ is considered highly risky for human health, in particular if coupled with other pollutants; a level below $100 \mathrm{~Bq} / \mathrm{m}^{3}$ is a safety level. As it appears from the table above, the Radon-222 $(\mathrm{Rn} 222)$ and its wide range of variability is the major responsible of the total level of radiation, and, at the same time, it is the only one which can be controlled and reduced by the human actions. On this basis, a set of recommendations (Table2) have been emanated in different countries and from ICRP (International Commission for Radio Protection), EPA (Environmental Protection Agency) and EU (Commission of the European Union), differentiating for existing and new buildings.

Table 2. Set of recommendations in different countries.

\begin{tabular}{lccccccccc}
\hline BUIIDING & Canada & USA & Sweden & U.K & Russia & Italy & ICRP & EPA & UE \\
\hline $\begin{array}{l}\text { EXISTTNG } \\
\begin{array}{l}\text { Level of } \\
\text { Action: }\end{array}\end{array}$ & 150 & 150 & 400 & 400 & $=$ & 400 & 400 & 150 & 400 \\
\hline $\begin{array}{l}\text { NEW } \\
\text { Design Level: }\end{array}$ & 150 & 150 & 140 & 100 & 140 & 150 & 100 & 100 & 200 \\
\hline
\end{tabular}

As far as the $\operatorname{Rn} 222$ sources are concerned, it is well known that these come from the Radium-226 decay; therefore all the materials rich of Radium-226 are significant sources of Rn222. If these materials made the soil below the buildings (mainly volcanic and granite soils and Al-rich clays) and/or the ceilings or the walls of the buildings, they would be responsible of the "indoor" radon concentration level. In order to have significant indoor accumulation of 
radon, it is not only necessary that the soil and/or the building materials are rich of Radium-226 but also that soil, building materials and building structures allow a significant flow of the Radon gas generated by Radium (by diffusive and convective transport phenomena) through them until it reaches, in a significant percentage, the building indoor space. The schematic flow of the whole process is represented hereinafter.

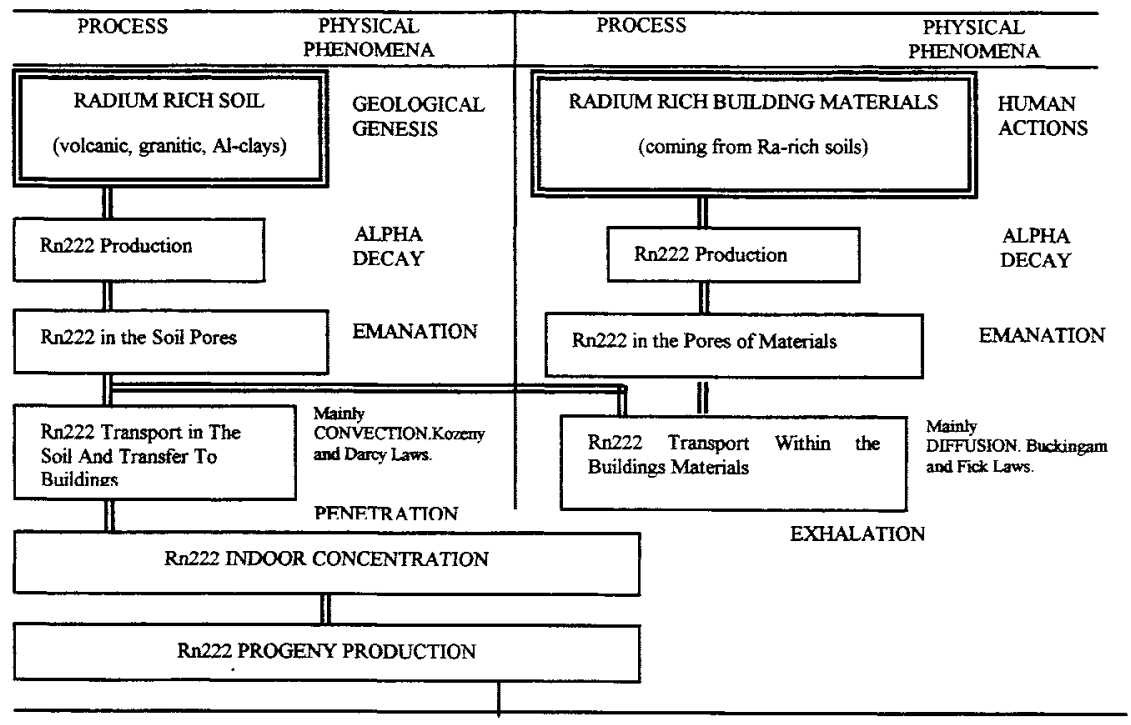

Figure 1: Flow of the whole process.

As far as the Radium- 226 content in building materials is concerned, hereinafter typical values for some common materials are listed: they clearly stress the different Radon production capability existing for different construction choices.

Table 3 . Radium-226 content in some common building materials.

\begin{tabular}{|l|c|}
\hline \multicolumn{1}{|c|}{ BULDING MATERIAL } & Ra-226 Content (Bq/kg) \\
\hline SAND & 0 \\
STONE & 90 \\
GYPSUM - calcium sulphate & 10 \\
CEMENT & 50 \\
CLAY -silicate of alumina & $50-120$ \\
BRICKS & 50 \\
POZZUOLANA & $190-380$ \\
TUFA (TUFF) & 160 \\
GRANITE & 180 \\
LAVA & $90-700$ \\
\hline
\end{tabular}

\section{Permeability and Kozeny's law}

In this paragraph the radon presence in the soil (and through it, in the building above the soil and in the building materials coming from the soil) is analysed as a function of the physical characteristics of the soil. For a given volume of soil, 
its percentage occupied by solid matter is called solid fraction, whereas the remaining percentage is named porosity $\varepsilon$ (ranging between 0 and 1 ), which can be filled by liquid (usually water) - humid component - or gas (usually atmospheric air). The types of soil are usually classified as clay, mud, sand, gravel. Humid component reaches the maximum values for clay and the minimum values for gravel. The main factor affecting the radon presence in the buildings is the soil permeability $\mathrm{k}$, as an index of the capability of the air to flow through the soil. It is measured in $\mathrm{m}^{2}$ and it is defined by the Kozeny Law:

$$
\mathrm{k}=\varepsilon^{2} \mathrm{~d}^{2}
$$

where $d$ is the average diameter of the solid particles constituting the soil. $K$ is linked to the fluid flows through the soil by the Darcy's Law (sec.5). The importance of the soil permeability derives from the wide range of values which it can exhibit. In fact, $\mathrm{k}$ ranges from values such as $10^{-8} \mathrm{~m}^{2}$ for gravel to $10^{-16} \mathrm{~m}^{2}$ for clay. The Rn222 gas can flow through the soil by means of molecular diffusion - in case of low permeability soil - or of convective flows - in case of high permeability soil. The presence indoor of $\mathrm{Rn}-222$ flowing into the buildings from the soil is mainly due to the ascensional convection in the soil (dominated by a pressure gradient existing in the soil), and therefore the Radon presence indoor is greater where the soil permeability is greater.

\section{Diffusivity and Buckingam's law}

The other source of Rn222 indoor is constituted by the building walls. The $\mathrm{Rn} 222$ coming to the rooms from the building walls is mainly due to the molecular diffusion in the building materials. The molecular diffusion is due to the presence of a concentration gradient exiting in the soil and it is regulated by the Fick's Law (sec.5). The concentration gradient of a fluid and the flow of the fluid in a material are linked by means of a diffusion coefficient $D\left(\mathrm{~m}^{2} / \mathrm{s}\right)$ when the whole volume occupied by a porous medium is considered, whereas an effective diffusion coefficient $\mathrm{D}_{\mathrm{e}}\left(\mathrm{m}^{2} / \mathrm{s}\right)$ is used when a single pore is considered. The two coefficients above are linked by the porosity $\varepsilon$ by means of:

$$
\mathrm{D}=\varepsilon \mathrm{D}_{\mathrm{e}}
$$

When the pore is represented by the "open air" (upper limit) the coefficient is called "open air diffusion coefficient $\mathrm{D}_{0}$ " $\left(\mathrm{m}^{2} / \mathrm{s}\right)$, and it represents the easiest coefficient to be measured. The relationship which better links the coefficient $\mathrm{D}$ of a certain medium having porosity $\varepsilon$ to the open air diffusion coefficient is given by the Buckingam Law:

$$
\mathrm{D}=\varepsilon^{2} \mathrm{D}_{0}
$$

As far as the Rn222 diffusion is concerned, the effective diffusion coefficient $D_{e}$ ranges from $2.2 \times 10^{-10}\left(\mathrm{~m}^{2} / \mathrm{s}\right)$ in the mud to $2.7 \times 10^{-6}$ in the sand, whereas the open air diffusion coefficent $D_{0}$ assumes the value of $1.2 \times 10^{-5}\left(\mathrm{~m}^{2} / \mathrm{s}\right)$.

\section{The emanation coefficient}

Only a percentage of the Rn222 generated in the soil or in the building walls is 
able to reach a pore, and, then, to reach the soil or the wall surface (and therefore the building indoor environment) by means of convective or diffusive phenomena. This percentage is called emanation coefficient $\mathrm{f}$, and it ranges in a wide interval, between $5 \%$ and $70 \%$. In fact, in the Radium-226 alfa-decay process, the $\mathrm{Rn} 222$ is generated with an initial kinetic energy of $86 \mathrm{Kev}$ ("recoil energy", due to the momentum conservation in the decay process). The so generated Rn222 atoms go through the medium ionizing or exciting the atoms and the molecules of the substances composing the medium, and loosing energy in any ionization or excitation process. The Radon migration process ends when it has lost all its initial energy; the distance that they are capable to cover in this time span typically are: $0.02-0.07 \mu \mathrm{m}$ in the common soil solid matter, $0.1 \mu \mathrm{m}$ in the water and $63 \mu \mathrm{m}$ in the air. It appears now that only the fraction of Rn222 generated at a distance smaller than 0.07 microns from a pore can reach the pore and enter the diffusive-convective loop described above. The moisture content strongly affects the emanation coefficient $\mathrm{f}$ : it increases almost exponentially when the moisture contents increases up to the condition where the moisture fills $25 \%$ of the pore volume; over this moisture value $f$ remains practically constant. All the above suggests that the radon delivery from the soil, due to high emanation coefficients in the soil and high convective-diffusive phenomena for the radon transport, is maximum when the soil has a medium moisture content, as it has in the most practical common circumstances. In fact, if the soil was sature of moisture, the emanation coefficient should be still high but diffusivity and permeability (which regulate the radon transport) should have very low values, whereas if the soil was completely dry, the emanation coefficient should be poor. As far as the temperature is concerned, it also affects the values of the radon emanation coefficient: $\mathrm{f}$-values are $55 \%$ greater when the temperature goes from 5 to $50^{\circ} \mathrm{C}$, and a linear interpolation can be roughly used to evaluate the f-value for intermediate temperatures.

\section{Radon in buildings: modeling and experimental results}

\subsection{Radon transport by means of diffusion. Fick's law}

In this section the mathematical approach describing the radon molecular diffusion (due to concentration gradients) and the radon convection (due to pressure gradients) in porous materials is introduced. Considering a gas made of two components $\mathrm{A}$ and $\mathrm{B}$, the molar flux of $\mathrm{A}$, with respect to a steady state reference system, is given by the first Fick's Law:

$$
N_{A}=x_{A} \cdot\left(N_{A}+N_{B}\right)-c \cdot D_{0} \cdot \operatorname{grad}\left(x_{A}\right)
$$

where $N_{A}$ and $N_{B}$ are the vectorial fluxes of $A$ and $B\left(\right.$ moles $\left./ \mathrm{m}^{2} / \mathrm{sec}\right), x_{A}$ is the $A$ molar fraction, $c$ is the gas molar concentration (moles $\left./ \mathrm{m}^{3}\right), \mathrm{D}_{0}$ is the diffusion coefficient $\left(\mathrm{m}^{2} / \mathrm{sec}\right)$. The first term in the right side of eqn (4) represents the flux of A due to convective phenomena whereas the second term gives the flux of A due to molecular diffusion with respect to the average velocity of the convective phenomena. In the case of Radon gas in air eqn (4) can be simplified as follows: 
1. the Radon molar fraction in air is neglect able and therefore the term $\mathrm{x}_{\mathrm{A}} \cdot \mathrm{N}_{\mathrm{A}}$ can be ignored with respect to $\mathrm{N}_{\mathrm{A}}$;

2. the air molar concentration can be assumed as a constant and therefore $c \cdot \operatorname{grad}\left(x_{A}\right)=\operatorname{grad}\left(c_{R n}\right)$, where $c_{R n}=c \cdot x_{R n}=$ Radon molar density.

In view of the approximations above, eqn (4) becomes:

$$
\mathrm{N}_{\mathrm{Rn}}=\mathrm{X}_{\mathrm{Rn}} \cdot \mathrm{N}_{\mathrm{Air}}-\mathrm{D}_{0} \cdot \operatorname{grad}\left(\mathrm{c}_{\mathrm{Rn}}\right)
$$

Multiplying eqn (5) by the Avogadro number and the Radon decay constant, the following equation is obtained:

$$
\mathrm{J}_{\mathrm{Rn}}=\mathrm{I}_{\mathrm{Rn}} \cdot \mathrm{V}_{\mathrm{Air}}-\mathrm{D}_{0} \cdot \operatorname{grad}\left(\mathrm{I}_{\mathrm{rN}}\right)
$$

where $J_{R n}$ is the activity of the Radon diffusive flux $\left(B q / \mathrm{m}^{2} / \mathrm{sec}\right), I_{R n}$ is the activity of the Radon concentration $\left(\mathrm{Bq} / \mathrm{m}^{3}\right), \mathrm{V}_{\text {Air }}$ is the net air velocity $(\mathrm{m} / \mathrm{sec})$. Furthermore, it must be considered that the Radon migration is within the soil, and not "open air"; thus, the area available for the Radon diffusion is reduced and, at the same time, the average path of a Radon atom is enhanced. These effects are taken into account substituting in eqn (6) the diffusion coefficient $\mathrm{D}_{0}$ with an effective diffusion coefficient $D_{\mathrm{e}}$. Therefore, the final eqn describing the radon diffusion/convection in porous materials will be:

$$
\mathrm{J}_{\mathrm{Rn}}=-\mathrm{D}_{\mathrm{e}} \cdot \operatorname{grad}\left(\mathrm{I}_{\mathrm{Rn}}\right)+\mathrm{I}_{\mathrm{Rn}} \cdot \mathrm{V}_{\text {Air }}
$$

In eqn (7) the following hypotheses are underlying:

- gas atoms interact among each other only (thus they don't interact with the pore surface)

- Radon gas is not adsorbed at all by the solid pore surface.

Eqn (7) is assumed as a basis for any kind of modelling activities regarding the gas Radon in the soil or in any porous solid material. The first term in the right side of eqn (7) represents the diffusive flux, while the second is the convective flux. Considering the diffusive flux only, the following eqn will follow:

$$
\mathrm{J}_{\mathrm{Rnd}}=-\mathrm{D}_{\mathrm{e}} \cdot \operatorname{grad}\left(\mathrm{I}_{\mathrm{Rn}}\right)
$$

Considering the vertical axis only, assuming that the radon concentration is zero on the soil surface and that the depth of the soil is infinite, it is obtained:

$$
J_{R n d}=\left(D_{e} \cdot \lambda_{R n}\right)^{1 / 2} \cdot \rho_{s} \cdot f \cdot A_{R a} \cdot(1-\varepsilon)
$$

where $\lambda_{R n}$ is the radon decay constant, $\rho_{s}$ is the soil density, $f$ is the emanation coefficient, $A_{R a}$ is the radium concentration activity in the soil, $\varepsilon$ is the soil porosity. Inserting in eqn (9) typical average values available for the Earth soil, it is possible to find $\mathrm{J}_{\mathrm{Rnd}}$ values such as:

$$
\mathrm{J}_{\mathrm{Rnd}}=0.016 \mathrm{~Bq} / \mathrm{m}^{2} / \mathrm{sec}
$$

Considering now that the building fundaments and basements are a further strong barrier for the radon diffusion (good fundaments and basements can reduce to $5 \%$ the radon diffusive flux values calculated above), it appears that:

- the radon diffusive flux is dominant on the radon convective flux for radon freely entering from the soil to the atmosphere (open air);

- vice-versa, the radon convective flux is definitely dominant on the radon diffusive flux for radon entering from the soil into the buildings (indoor). 


\subsection{Radon transport by means of convection. Darcy's law}

Following the considerations introduced in the last sentences of the par.5.1, the convective part of eqn (7) should be considered:

$$
\mathrm{J}_{\mathrm{Rnc}}=\mathrm{I}_{\mathrm{Rn}} \cdot \mathrm{V}_{\mathrm{Air}}
$$

If the soil permeability is constant and isotrope, the velocity $\mathrm{V}_{\text {Air }}$ is given by the following Darcy's Law:

$$
\mathrm{V}_{\mathrm{Air}}=-(k / \mu) \cdot \operatorname{grad}(\mathrm{P})
$$

where $\mathrm{k}$ is the intrinsic soil permeability, $\mu$ is the fluid viscosity and $\operatorname{grad}(\mathrm{P})$ is the pressure gradient. The convective radon flux towards the indoor of a building is proportional to the pressure gradient existing between the building indoor space and the pressure of the interstitial air in soil, fundaments, basement and walls. This pressure gradient is usually well represented by expressions of the following type:

$$
\Delta(\mathrm{P})=\mathrm{a} \cdot \Delta \mathrm{T}+\mathrm{b} \cdot \mathrm{w}^{2}
$$

where:

- $\quad \mathrm{a}$ " and "b" are two coefficients depending on the house typology and shape, and on the number and type of openings and fractures existing in the building

- $\Delta \mathrm{T}$ is the difference of temperature existing between the indoor ambient and the considered external ambient $\left({ }^{\circ} \mathrm{K}\right)$

- $\quad w$ is the velocity of the air movement (ventilation) in the house $(\mathrm{m} / \mathrm{sec})$.

Substituting eqns (11) and (12) into eqn (10) and considering the vertical axis only, it is obtained:

$$
\mathrm{J}_{\mathrm{Rnc}}=-\mathrm{I}_{\mathrm{Rn}} \cdot(\mathrm{k} / \mu) \cdot\left[\mathrm{a} \cdot \Delta(\mathrm{T})+\mathrm{b} \cdot \mathrm{w}^{2}\right]
$$

The analysis of the structure of eqn (13) lead to the following considerations confirmed by many experimental measurements:

(A) In general terms, the indoor concentration of Rn-222 is strongly influenced not only by the Ra concentration in the underlying soil and in the building materials but also by a combination of physical, structural and meteorological parameters such as: temperature, humidity, soil porosity and presence of openings and fractures in the building lower parts, wind, rain and variations of the atmospheric pressure.

(B) Looking at the term containing $\mathrm{w}^{2}$, it appears that the indoor concentration of $\mathrm{Rn}-222$ is strongly influenced by the square of the ventilation rate, falling down when $\mathrm{w}$ goes up. Furthermore, if the ventilation includes air exchange between the indoor and the outdoor of the building, the effect is even more evident because the radon present indoor is continuously shifted outdoor. The experimental data confirm that for a Rn-222 activity concentration values of the order of $40-50 \mathrm{~Bq} / \mathrm{m}^{3}$ (typical of normal buildings), a reduction of the air exchange from 1 air/exchange/hour to 0.3 air exchange/hour determines an increase in the Working Level Months values from WLM $=0.081$ to 0.407 (some 5 times greater!). As a consequence, the Rn-222 activity concentration is also a function of the seasons; its summer values are normally lower than the corresponding winter values because of the usual habit to maintain windows and 
doors closed in winter and open in summer, unless an air conditioning system is adopted in summer, which in any case contributes to the radon reduction because of the higher $\mathrm{W}$ values induced in the rooms.

(C) Looking at the term of eqn (13) containing $\Delta \mathrm{T}$, for purely theoretical building having walls and roof made of impenetrable steel, hermetically closed all around, when the temperature indoor is higher than the temperature in the soil, the pressure indoor is greaten than in the soil and no convection should happen. As the soil temperature, at least in the first 3-6 meters of depth, is some equal to the yearly average temperature of the site, it follows that only in winter a Radon convective flux can take place from the soil to the building and even in winter it would not happen if an heating system is switched on in the house to bring the indoor temperature to values higher than the yearly average temperature of the building site. In real cases, due to the natural infiltrations of air into the building through the walls, windows and openings connecting the house to the outdoor environment, and due to the air thermal stratification in the rooms, when the indoor temperature increases the air flow from the indoor space to the open air space increases as well: this fact generates low pressure values inside the building (chimney effect) capable to extract much more air (and radon gas) from the coldest neighbouring areas (for example from soil or walls). In fact the same eqns above are valid to regulate the heat and mass transfer phenomena between the rooms and the outdoor open space. Thus, the final effect normally is that the increase of temperature in a building generates higher values of $\mathrm{Rn}-222$ activity concentration inside the building itself. This is confirmed by the analysis of the results of the available experimental measurements which are summarized and represented in Figures 2 and 3. These figures represent the $\mathrm{Rn}-222$ exhalation rate ER (atoms $/ \mathrm{m}^{2} / \mathrm{s}$ ) and the Rn-222 activity concentration AC $\left(\mathrm{Bq} / \mathrm{m}^{3}\right)$ as a function of the temperature $\mathrm{T}$ (in ${ }^{\circ} \mathrm{C}$ ), indicating also the spread of the available measurement results and the relevant correlation factors.

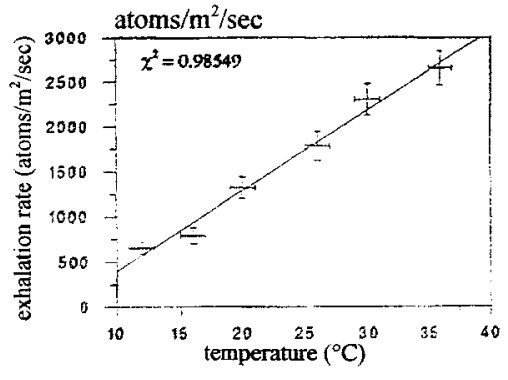

Figure 2: Rn-222 exhalation rate vs building indoor temperature.

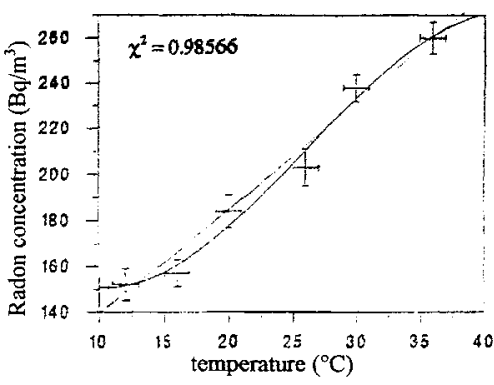

Figure 3:Rn-222 Indoor Activity Concentration vs building indoor temperature.

The eqns correlating the experimental data and representing the curves in the figures are: $\mathrm{ER}=84.74 \cdot \mathrm{T}-448.43 ; \quad \mathrm{AC}=0.09 \cdot \mathrm{T}^{2}+140.72$ 


\section{$5.3 \mathrm{Rn}-222$ transport through building materials}

The $\mathrm{Rn}-222$ Concentration in a room derives from three main factors:

1) The quantity of Radon produced and directly coming in/from the soil by means of the diffusive-convective processes (convection prevalent). This factor:

- is usually highly dominant in the basement floor of the buildings

- depends exclusively on type and composition of the soil below the building as well as on the type and quality of the buildings fundaments.

2) The quantity of Radon produced in the soil and coming to the rooms passing through the building walls by means of analogous diffusive-convective processes (diffusion prevalent) seen above. This factor:

- is present and can contribute to the room's AC values of the basement and (in a minor percentage) of the 1st floor (if basement is not so high);

- depends on the soil composition as well as on the building wall type, composition and thermohygrometric conditions (which affect the walls' exhalation rate).

3) The quantity of Radon produced in the building walls and exhalated from the walls to the rooms. This factor:

- is practically the sole factor responsible of the Radon concentration in the upper floors of the building (from the 1st floor to the roof);

- depends exclusively on the quantity of Radium $\left(\mathrm{Ra}_{88}^{226}\right)$ present in the walls and therefore on the walls prime materials and structural organization (which affect the walls' exhalation rate).

As far as the last factor is concerned, many authors estimate the $\mathrm{Rn} A C$ value in the rooms due to the Radium present in the walls through formulas like:

$$
\mathrm{AC}_{\mathrm{Rn}}=(\mathrm{ER} \cdot \mathrm{S}) /(\mathrm{V} \cdot \mathrm{VR})
$$

where $S$ and $V$ are respectively the walls' surface and the room volume, VR is the room ventilation rate (number of air exchange/hour) and ER is the $\mathrm{Rn} 222$ Exhalation Rate (number of atoms $/ \mathrm{hour} / \mathrm{m}^{2}$ ). In the eqn (14), the values of ER are estimated through the following relationship, taking into account the sole diffusive phenomena in the walls (being the convection neglectable because of the usual low permeability of the walls' materials):

$$
\mathrm{ER}=\mathrm{AS}_{\mathrm{Ra}} \cdot \lambda_{\mathrm{Rn}} \cdot \rho \cdot \mathrm{f} \cdot \mathrm{d} / 2
$$

where $\mathrm{AS}_{\mathrm{Ra}}$ is the Radium-226 Specific Activity $(\mathrm{Bq} / \mathrm{kg})$ in the wall materials, $\lambda_{R n}$ is the Rn222 decay constant, $\rho$ is the density of the wall materials $\left(\mathrm{kg} / \mathrm{m}^{3}\right), \mathrm{f}$ the emanation rate and $\mathrm{d}$ is the wall thickness $(\mathrm{m})$.

\subsection{A set of experimental results}

All the considerations above have been confirmed and validated during a campaign of measurements carried out in Central-Southern Italy. Figures 4, 5, 6 summarize some of the most significant results regarding a thousand of measurements carried out on some hundreds of buildings, tested at three levels (underground level, ground floor and 1st floor) and exhibiting different construction materials and building techniques ("tufa", concrete and bricks). 


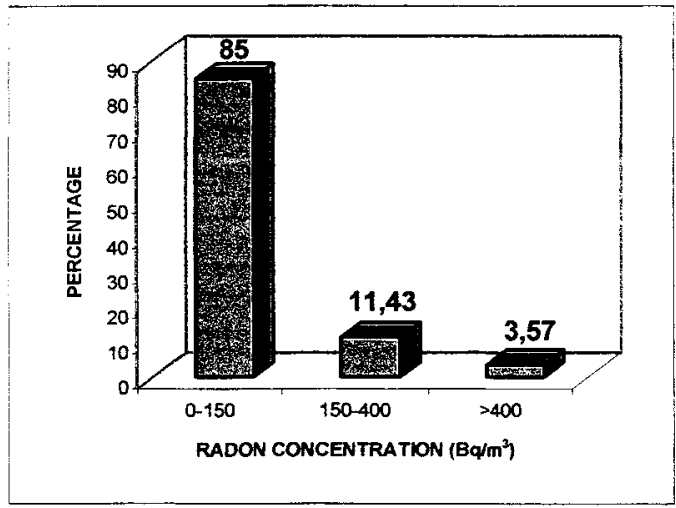

Figure 4: Percentage distribution of the radon concentrations in Catanzaro dwellings according to reference levels recommended by EPA and ICRP.

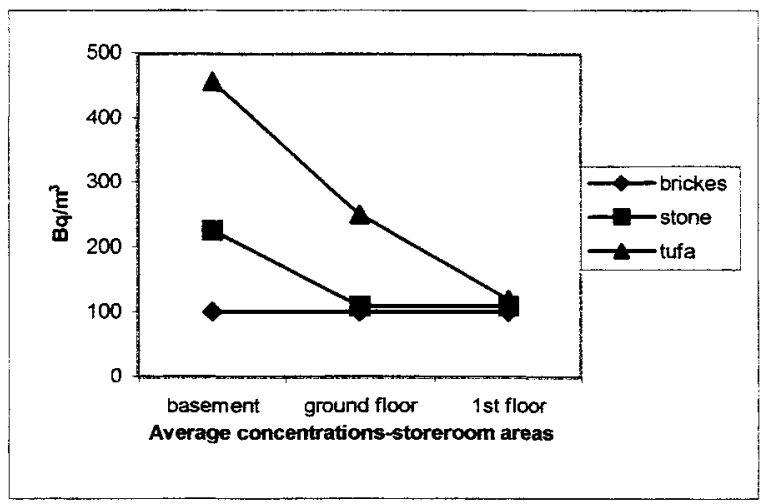

Figure 5: Average radon concentrations distributions in the storeroom areas at different building floors.

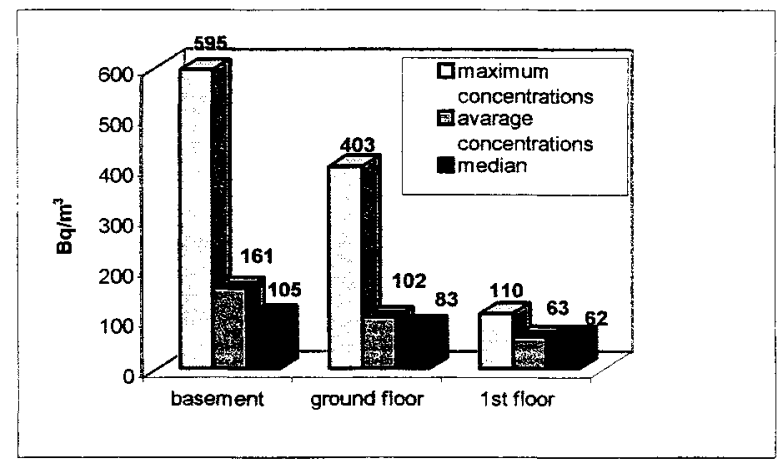

Figure 6: Distribution of the maximum, average and medium concentrations at different building floors. 